\title{
JUAN DEL ENCINA (1469-1529) \\ UND DIE BEDEUTUNG DES HUMANISMUS FÜR DIE SPANISCHE MUSIK AM ENDE DES 15. JAHRHUNDERTS
}

Michael ZYWIETZ

\begin{abstract}
In recent decades studies on humanism have stressed the significance of the humanist movement in Spain during the $15^{\text {th }}$ and $16^{\text {th }}$ centuries and thus countered the dictum of "cultural belatedness" or the absence of a Spanish Renaissance. According to education and activity Encina must be called a humanist; moreover he personifies an ideal by combining the functions of poet and composer. In the Cancionero Musical de Palacio Encina is clearly the dominating composer. Since the cultivation of music at the court of the catholic kings in the second half of the $15^{\text {th }}$ century was comparable to that at other European courts, it is surprising that Spanish composers took up the international style, dominated by franco-flemish composers, only in a very limited way, but established a separate national tradition in the field of secular vocal music. The striking simplicity of Spanish secular polyphony has often been interpreted as a deficiency and the lack of ability to adapt the compositional techniques of franco-flemish composers, who were taken as stylistic model; whereas the Spanish music historiography of the $19^{\text {th }}$ and early $20^{\text {th }}$ century emphasizing national aspects endeavored to show equivalents -nonexistent in such a way - to developments in Burgundy and Italy. Taking Encinas "Levanta Pascual" as example, the response to a concrete historical event (the fall of Grenada 1492) characteristic of a specific humanist conception will be illustrated.
\end{abstract}

\section{Zusammenfassung}

In den jüngst vergangenen Jahrzehnten hat dir Humanismus-Forschung die Bedeutung von der humanistischen Bewegung in Spanien im 15. und 16. Jahrhundert hervorgehoben und ist so dem Diktum der „kulturellen Verspätung” und dem Fehlen einer Renaissance in Spanien entgegengetreten. Nach Ausbildung und Tätigkeit ist Encina als Humanist zu bezeichnen, zudem verkörpert er das Idealbild der Personalunion von Dichter und Komponist. Im Cancionero Musical de Palacio ist Encina eindeutig dominierende die. Komponistenpersönlichkeit Da die Musikpflege am Hofe der Katholischen Könige in der 2 Hälfte des 15. Jahrhunderts auf einer vergleichbaren Höhe mit der anderer europäischer Höfe stand und das dort gepflegte Repertoire international ausgerichtet war, verwundert es, daß die spanischen Komponisten sich den durch die frankoflämischen Komponisten dominierten internationalen Stil nur in sehr begrenzter Art und Weise aneigneten und stattdessen auf dem Gebiet der weltlichen Vokalmusik eine nationale Sondertradition begründeten. Die auffällige Einfachheit der weltlichen Polyphonie Spaniens ist häufig als Zurückgebliebenheit und mangelnde Fähigkeit zur künstlerischen Adaption der als Stilmaßstab verstandenen Kompositionstechnik der frankoflämischen Komponisten interpretiert worden; wohingegen eine den nationalen Aspekt betonende spanische Musikgeschichtsschreibung im 19. Jahrhundert und in der ersten Hälfte des 20. Jahrhunderts um den Nachweis einer, so nicht existierenden, Gleichberechtigung mit den Entwicklungen in Burgund und Italien bemüht war. Am Beispiel von Encinas „Levanta, Pascual” wird das von spezifisch humanistischen Auffassungen geprägte Eingehen auf ein konkretes historisches Ereignis - den Fall Granadas 1492 - dargelegt.

Anuario Musical, 57 (2002) 
In seinem grundlegenden Werk The Rise of European Music bezeichnet Reinhard Strohm einen Vergleich der Musik Juan del Encinas mit der italienischen Frottola und seiner Poesie mit der von Agnolo Poliziano und Jacopo Sannazaro als ein Desiderat zukünftiger Forschung. ${ }^{\prime}$ Da dieser Anregung, soweit ich sehe, bis heute niemand nachgegangen ist, soll hier im folgenden an einem Beispiel eine derartige Gegenüberstellung versucht werden. Zugleich wird jenem Einfluß nachgegangen, den die spezifisch spanische Ausprägung des Humanismus auf die Musik des Landes ausübte. $\mathrm{Zu}$ diesem Zwecke werden in einem ersten Schritt Grundtendenzen des spanischen Humanismus im 15. Jahrhundert erörtert. Sodann werden der Forschungsstand zu Encina und zum Cancionero Musical de Palacio ${ }^{2}$ (im folgenden CMP) dargestellt und kommentiert. In einem vierten Abschnitt erfolgt die Anwendung der Ergebnisse auf den konkreten Gegenstand der Betrachtung.

\section{Der spanische Humanismus im 15. Jahrhundert}

Gerade unter dem von der gegenwärtigen Forschung mit großer Aufmerksamkeit bedachten Aspekt der Patronage ist die tatkräftige Förderung humanistischer Kultur durch die Königin Isabella von besonderem Interesse. Ihrem Beispiel folgend und wohl auch aus eigenem Antrieb - auf die fundamentale Bedeutung des antiken Topos der , armas y letras ' für die spanische Kultur des siglo de oro kann hier nur hingewiesen werden ${ }^{3}$ - bildeten Adelige eigene literarische Höfe und trugen große Bibliotheken zusammen. Stellvertretend für viele sei auf den Marqués de Santillana hingewiesen, der mit seinem Proverbios (1437) die wichtigste Poetik des 15. Jahrhunderts in kastilischer Sprache vorlegte und dessen Bibliothek ein Zentrum des frühen Humanismus in Kastilien ist. Der zweite große Dichter der Epoche Juans II. (1406-1454) ist Juan de Mena (1411-1456), dessen episches Hauptwerk El Laberinto de la Fortuna (1444) deutlich das Vorbild Vergils und Lucians erkennen läßt und zugleich in offenen Wettstreit mit den Dichtern der Antike tritt. Dieser Zwiespalt von Nachahmung und Konkurrenz ist ein typisches Charakteristikum des Humanismus. Ein weiterer Ausdruck humanistischer Bestrebungen ist die im 15. Jahrhundert einsetzende intensive Übersetzertätigkeit, die sowohl die wesentlichen Werke der griechischen und römischen Antike als auch die der modernen Literaturen Italiens, Frankreichs und Englands ins Kastilische übertrug. ${ }^{4}$

Im Jahr der Wiedereroberung Granadas legte Antonio de Nebrija (1444-1522), einer der Lehrer Juan del Encinas an der Universität von Salamanca, mit seinem Diccionario latino-espanol nicht nur die erste spanische Grammatik vor, sondern die einer romanischen Sprache überhaupt. Erklärte

1. Vgl. Reinhard Strohm, The Rise of European Music, 1380-1500. Cambridge 1993, S. 580. „To compare his (Encinas) music with that of the frottolists, and his poetry with that of Poliziano and Sannazaro, seems an interesting task of the future."

2. Madrid. Palacio Real, Biblioteca. MS 1335 (olim 2-I-5). Vgl. hierzu Census-Catalogue of Manuscript Sources of Polyphonic Music 1400-1550, Bd. II, Neuhausen-Stuttgart 1982, S. 135f.

3. Weitere Literatur zu diesem Themenkomplex: Nicholas G. Round, Renaissance Culture and ist Opponents in Fifteenth-Century Castile. In: Modern Language Review 57, 1962, S. 204-215; P. E. Russell, Las armas contra las letras: para una definición del humanismo español del siglo XV. In: ders., Temas de „La Celestina“ y otros estudios, Barcelona 1978, S. $207-239$.

4. Vgl. Karl Kohut, Das 15. Jahrhundert. In: Geschichte der spanischen Literatur, hrsg v. Christoph Strosetzki. Tübingen 1991, S. 35-83. Hier: S. 44, Fußnote 11.

60

Anuario Musical, 57 (2002)

(c) Consejo Superior de Investigaciones Científicas Licencia Creative Commons 3.0 España (by-nc) 
Absicht Nebrijas war es, das Spanische zu einer Kunstsprache zu machen, die den großen Sprachen der Antike ebenbürtig sein sollte.

Im Hinblick auf die dichterische und musikalische Form des Villancico ist die Tatsache von besonderer Bedeutung, daß der Humanist Nebrija auch die volkstümlichen Formen der Dichtung für überlieferungswürdig erachtet. Nach dem Urteil von Karl Kohut bezeichnet seine Grammatik ,eine Zeitenwende, die das späte Mittelalter von der neuen Zeit des Humanismus trennt. “5

Offensichtlich besaßen bereits die Zeitgenossen ein Bewußtsein dafür, daß mit Santillana und Juan de Mena eine neue Ära begonnen habe, zuvor hatte aber bereits Inigo López de Mendoza (13981458) im ,Proemio“ zu El triunfo de Marqués (um 1459) behauptet, daß die Beredsamkeit Italien verlassen habe und nach Kastilien gegangen sei, wo sie nun die Italiener in kastilischer Sprache übertreffe. $^{6}$

Ohne die durch den Gebrauch der Begriffe Mittelalter und Renaissance entstehenden Probleme mit einer diesen Begriffen impliziten und so nicht existenten Zäsur zu verkennen, betont Christoph Strosetzki nachdrücklich die Bedeutung und Eigenart des Humanismus in Spanien. ${ }^{7}$ Ungeachtet dieser Tatsache wurde in unserem Jahrhundert ein allegorisches Lehrwerk über die artes liberales, die Vision delectable des Alfonso de la Torre (entstanden in den Jahren 1430 bis 1440) zum Anlaß einer Polemik über die „kulturelle Verspätung Spaniens“ und die Existenz einer Renaissance in Spanien. ${ }^{8}$

Es ist hier nicht der Ort, eine eingehende Diskussion der Thesen von Ernst Robert Curtius zu unternehmen, doch ist festzuhalten, daß die von ihm als Ausdruck generellen Zurückbleibens gewertete verspätete und dann auch nicht sehr ausgeprägte Entwicklung des Feudalstaates in Spanien von der Geschichtswissenschaft heute kaum mehr ausschließlich negativ bewertet wird. ${ }^{9}$ Weiterhin gibt es zu denken, daß Curtius seine weitreichenden Thesen allein auf das genannte Werk von Torres gründet, ${ }^{10}$ ohne die oben erwähnten nahezu zeitgleichen Werke Santillanas, Menas u. a. zu

5. K. Kohut, a. a. O., Fußnote 4, S. 47. Grundlegend zur Problematik der Abgrenzung des Mittelalters von der Renaissance ist nachwievor August Buck, Das Geschichtsdenken der Renaissance (= Schriften und Vorträge des Petrarca-Instituts Köln, Bd. IX). Krefeld 1957.

6. Vgl. Jeremy N. H. Lawrance, Humanism in the Iberian Peninsula. In: The Impact of Humanism in Western Europe, hrsg. v. Anthony Goodman und Angus MacKay. London und New York 1990, S. 220-258. Hier: S. $220 \mathrm{f}$.

7. Vgl. Christoph Strosetzki, Literatur als Beruf. Zum Selbstverständnis gelehrter und schriftstellerischer Existenz im spanischen Siglo de Oro (= Studia humaniora, Bd. 8). Düsseldorf 1987, S. 7.

8. Vgl. Ernst Robert Curtius, Spaniens kulturelle „Verspätung“. In: Ernst Robert Curtius, Europäische Literatur und lateinisches Mittelalter. Bern und München 1965 (5. Auflage, 1. Auflage 1948), S. 524-526. In einem Aufsatz aus dem Jahre 1927 glaubt Victor Klemperer die von ihm gestellte Frage nach der Existenz einer Renaissance in Spanien verneinen zu können: „Was nun auf Italien, Frankreich, Deutschland und England zutrifft, daß sie als neue europäische Völker längst „geboren“waren, daß also ihre Volksbestandteile (Lateiner und Germanen in Italien, Slaven (sic!) und Germanen in Deutschland, usw., usw.) sich physisch und geistig zusammengefunden hatten, und weiter, daß seit der „Geburt“ eine Zeit verflossen war, in der ruhige Reife und Ueberreife des Kirchlichen hatte eintreten können: genau diese beiden die Renaissance vorbedingenden Punkte treffen auf Spanien nicht zu. Es hat ein außereuropäisches Schicksal erlitten. So eng es der lateinischen Sprache und der römischen Kirche verknüpft, und so viel gotisches Blut ihm zugeflossen ist: es gehört nur halb zu Europa. Afrika hat einen gewaltigen Anteil an der iberischen Halbinsel, ehe sie romanisiert wird." Victor Klemperer, Gibt es eine spanische Renaissance? In: Logos. Internationale Zeitschrift fiir Philosophie der Kultur, XVI (1927), S. 129-161. Hier: S. 139-140.

9. Die von Curtius herangezogene historische Reverenzquelle war bereits zum Zeitpunkt des Erscheinens der ersten Auflage seines grundlegenden Werkes vom Standpunkt der historischen Forschung aus betrachtet als veraltet zu bezeichnen. Vgl. Claudio Sanchez-Albornoz, Espana y Francia en la Edad Media. Causas de su diferenciación política. In: Revista de Occidente 2 (1923), S. 294-316.

10. „Alfonso de la Torre ist, obwohl er im 15. Jahrhundert schrieb, fast unberührt sowohl von der lateinischen Scholastik des 13. Jahrhunderts wie vom echten Aristotelismus. Was er dem Spanien des 15. bis 17. Jahrhundert vermittelt, ist eine eklektische 
berücksichtigen. Neben diesen Zeugnissen spricht etwa auch folgende, zu Encina in direkter Beziehung stehende Tatsache, gegen die Annahme einer „kulturellen Verspätung“ Spaniens. So weist Paul Oskar Kristeller anhand von Dokumenten, die den Musikunterricht an den italienischen Universitäten belegen, darauf hin, daß der Spanier Bartolomé Ramos de Pareja (um 1440 - um 1500), als er im Jahre 1482 seine Musica practica in Bologna veröffentlichte, zwar als öffentlicher Professor der Musik bezeichnet wurde, ihm diese Stelle jedoch nur versprochen worden war und er schon im nächsten Jahr Bologna wieder verließ, weil er sie niemals erhalten hatte. Zwar gibt es einen Erlaß von Papst Nikolaus V. aus dem Jahre 1450, der die Einrichtung eines Lehrstuhles für Musik in Bologna vorsah, aber es existieren keine Dokumente, die beweisen, daß dieser Lehrstuhl in den folgenden Jahren jemals besetzt wurde. Ramos de Pareja hatte aber vor seiner Italienreise in Salamanca an der Universität, an der später Encina Jurisprudenz studierte, Vorlesungen über Musik gehalten. ${ }^{11}$ Ohne diesen Aspekt überbewerten zu wollen, haben wir es hier doch mit einem Hinweis zu tun, der gegen ein Zurückgebliebensein im Sinne von Curtius spricht. Ramos de Parejas Musica practica dürfte zudem auch die Summe seiner Lehrerfahrungen an der Universität in Salamanca reflektieren und nicht nur die in Italien empfangenen Eindrücke wiedergeben. In seiner Wendung gegen die beiden wichtigsten musikalischen Autoritäten des Mittelalters, Boethius und Guido von Arezzo, dokumentiert sich eine der fortschrittlichsten Positionen seiner Zeit. ${ }^{12}$

Ein derartiges Eingehen auf die Position von Curtius mußte an dieser Stelle vor allem deswegen erfolgen, weil sie, wie weiter unten zu zeigen sein wird, die musikgeschichtliche Bewertung der Entwicklungen in Spanien wesentlich beeinflußt zu haben scheint. Richtig ist, daß die historischen Wurzeln des spanischen Humanismus in der Tat weit in die Zeit des Mittelalters zurückreichen. Die Eroberungen im byzantinischen Osten unter Jakob II. (1286-1327), die zur Gründung der Herzogtümer von Athen und Neopatras führten, der ständige Kontakt mit den geistigen und kulturellen Strömungen, die am päpstlichen Hof in Avignon zusammenliefen und die politischen und künstlerischen Kontakte mit Sizilien und Neapel führten zu einer intensiven Erforschung und Übersetzung der griechischen und lateinischen Klassiker bereits am Hofe Johanns I. (1387-1395). ${ }^{13}$ Der Hof Alfonsos V. in Neapel war eines der führenden Zentren des Humanismus in Europa. ${ }^{14}$

Verarbeitung von Wissensstoff, der teils auf Spätantike und Vormittelalter (Martianus Capella und Isidor), teils auf die lateinische Renaissance des französischen 12. Jahrhunderts (Alanus), teils auf den häretischen Aristotelismus der jüdischen und arabischen Denker des spanischen 12. Jahrhunderts zurückgeht." E. R. Curtius, a. a. O., Fußnote 8, S. 525.

11. Vgl. Paul Oskar Kristeller, Musik und Gelehrsamkeit in der frühen italienischen Renaissance. In: Humanismus und Renaissance, 2 Bde., hrsg. v. Eckhard Keßler. München o. J., 2. Bd., S. 149-163. Hier: S. 152. In Salamanca disputierte Ramos auch mit Pedro de Osma, Professor der Theologie an der Universität von Salamanca, über die drei griechischen Genera. Vgl. hierzu den Kommentar von Clement A. Miller in: Bartolomeo Ramis de Pareia, Musica Practica. Commentary and Translation by Clement A. Miller (=Musicological Studies and Documents, Bd. 44). Neuhausen-Stuttgart 1993, S. 16.

12. Clement A. Miller (vgl. Fußnote 11) spricht vom „,revolutionary and rather provacative character" des Traktates, ebda., S. 18. Vgl. auch Higino Anglés, La música en la Corte de los Reyes catolicos (= Monumentos de la Mùsica Española, Bd. I). Barcelona 1960 (2. Auflage, 1. Auflage 1941). Kapitel I.13: Bartolomé Ramos de Pareja y la enseñanza musical en la universidad de Salamanca, S. 40-43.

13. Vgl. Miguel Batllori, Joan-Luís Vives in der Geschichte der aragonisch-katalanischen Renaissance. In: Juan Luis Vives. Arbeitsgespräch in der Herzog August Bibliothek Wolfenbiittel vom 6. bis 8. November 1980, hrsg. v. August Buck (=Wolfenbütteler Abhandlungen zur Renaissanceforschung, Bd. 3). Hamburg 1981, S. 71-80. Hier: S. 73. Ders., Der katalanischaragonische Humanismus vom 14. bis 16. Jahrhundert (= Vorträge der Aeneas-Silvius-Stiftung an der Universität Basel, Bd. 12). Basel und Stuttgart 1977. Hier: S. 5.

14. Zur Musikpflege am Hof in Neapel und den Verbindungen nach Spanien vgl. Allan W. Atlas, Music at the Aragonese Court of Naples. Cambridge 1985. Passim. 
Insbesondere der direkte Kontakt zu Griechenland trug dazu bei, daß der spanische Humanismus zunächst ein ausgeprägt griechisches Aussehen annahm.

Ein wesentlicher Aspekt eines spezifisch spanischen Humanismus besteht zudem in der durch die Jahrhunderte konstanten Rezeption der Werke Senecas, die wesentlich unter den Vorzeichen des nationalen Selbstbewußtseins zu betrachten ist. ${ }^{15}$ Die Inanspruchnahme Senecas als eines klassischen Repräsentanten spanischer Kultur führte im 15. Jahrhundert zu einer gegen Italien gerichteten Identifikation der spanischen Philosophie mit dem Stoizismus in seiner Ausprägung durch Seneca, dem die vermeintlich für Italien bezeichnende Philosophie Epikurs, in ihrer zeitgenössischen Ausprägung durch Lorenzo Valla, entgegengestellt wurde. Als Beleg für die frühe Vertrautheit mit den Strömungen des italienischen Humanismus mag die Tatsache gelten, daß die erste vollständige und in Terzinen verfaßte Übersetzung der Divina Commedia, die in Europa entstand, von dem katalanischen Humanisten Andreu Febrér um 1428/29 angefertigt wurde. ${ }^{16}$

Wie wichtig der personelle Austausch für die Vermittlung kultureller Errungenschaften ist, hat die Forschung in den letzten Jahren verstärkt herausgestellt. Aufschlußreich sind die bei Peter Burke mitgeteilten Listen, die den Aufenthalt von Italienern in anderen Ländern und den von ausländischen Humanisten in Italien belegen. So wurden im 15. Jahrhundert etwa Pietro de Martire d'Anghiera und Lucio Marineo durch adelige Mäzene zu einem Aufenthalt in Spanien aufgefordert. Angelo Decembrio reiste um das Jahr 1458 nach Spanien, um dort nach Manuskripten für seine Arbeit zu suchen. ${ }^{17}$

Speziell für die Ausbildung von Spaniern in Italien gründete der spanische Kardinal Albornoz im Jahre 1364 in Bologna ein Kolleg. Dort erhielt neben Nebrija, dem Lehrer Encinas, auch Sepulveda seine Ausbildung. ${ }^{18}$ Hier, wie auch im Einfluß der spanischen Päpste und aufgrund der Tatsache, daß um 1500 von den 60.000 Einwohnern Roms 10.000 Spanier waren, ${ }^{19}$ eröffnen sich für die zukünftige Forschung noch wichtige Forschungsgebiete im Hinblick auf den kulturellen Austausch zwischen Italien und Spanien.

\section{Der Dichterkomponist Juan del Encina}

Versteht man unter einem Humanisten jenen Typus von Gelehrten, der sich professionell und aus Berufung mit den studia humanitatis - an einer Reihe von zeitgenössischen Dokumenten ist der Kanon der humanistischen Disziplinen genau ablesbar: Grammatik, Rhetorik, Poesie, Geschichte und

15. Vgl. Karl Alfred Blüher, Seneca in Spanien. Untersuchungen zur Geschichte der Seneca-Rezeption in Spanien vom 13. bis 17. Jahrhundert. München 1969. Hier: S. 169ff. und passim. Zur Bedeutung des Stoizismus für die spanische Geisteskultur vgl. auch Christoph Strosetzki, Grammatiker, Humanisten und Moralisten. In: Ch. Strosetzki (Hg.), Geschichte der spanischen Literatur, a. a. O., Fußnote 4, S. 214-225. Hier: S. 214 f.

16. Vgl. J. N. H. Lawrance, a. a. O., Fußnote 6, S. 246.

17. Vgl. Peter Burke, The Spread of Italian Humanism. In: The Impact of Humanism on Western Europe, hrsg. v. Anthony Goodman and Angus MacKay. London und New York 1990, S. 1-22. Hier: S. 5 und S. 7-16

18. Vgl. Gilbert Chase, Juan del Encina: Poet and Musician. In: Music \& Letters XX (1939), S. 420-430. Hier: S. 420. Vgl. weiterhin P. O. Kristeller, a. a. O., Fußnote 11, Bd. 2, S. 88.

19. Vgl. Michael Rössner, Das Theater des Siglo de Oro. In: Ch. Strosetzki (Hg.), Geschichte der spanischen Literatur, a. a. O.. Fußnote 4, S. 161-191. Hier: S. 168. 
Moralphilosophie, zu denen noch das Studium der griechischen und römischen Autoren der klassischen Antike hinzuzurechnen ist - beschäftigt, und unter Humanismus jene Literatur, Gelehrsamkeit und Gedankenwelt, die in den Schriften der Humanisten zum Ausdruck kommen, so ist Encina mit allem Recht als ein Humanist zu bezeichnen. Dichterkomponisten wie Guillaume de Machaut (um 1300-1377), Francesco Landini (um 1325-1397) und eben Encina antizipieren historisch das bei Gioseffo Zarlino formulierte und am Vorbild der Antike orientierte Idealbild der Einheit von Poet und Komponist. ${ }^{20}$

Dies beginnt bereits bei dem von ihm angenommenen Humanistennamen. Im Spanischen bezeichnet das Wort encina die Eiche, jenen Baum, der bei Vergil als der geheiligte Baum Jupiters begegnet. ${ }^{21}$ An der Universität von Salamanca, an der Encinas Bruder Diego de Fermoselle von 1478 bis 1522 Musik unterichtete, erwarb Encina einen Universitätsabschluß in Jurisprudenz. ${ }^{22}$ Zwar fehlen Zeugnisse wie das Del Lagos (1541) aus Italien, die einen engen Bezug von Text und Musik fordern, ${ }^{23}$ für Spanien im allgemeinen und für Encina im besonderen, doch läßt sich an Encinas Arte de poesía castellana, die zuerst zusammen mit Encinas Cancionero (1496) - der alle seine Werke umfaßte, die er zwischen seinem vierzehnten und fünfundzwanzigsten Lebensjahr verfaßt hatte - erschien, unschwer ein intensives Bemühen um eine Sprachkultur ablesen, die sich selbst an einem Punkt der Vollkommenheit angelangt sieht. Zum Zeitpunkt der Veröffentlichung des CMP erfreute sich Encina der Patronage von Fadrique Álvarez de Toledo, dem zweiten Herzog von Alba und lebte an dessen Hof in Alba de Tormes, widmete die Sammlung aber nicht diesem, sondern den Katholischen Königen.

Die im Cancionero enthaltenen Werke seiner frühesten Schaffensphase sind in Metrum und Vokabular ganz der kastilischen Tradition verhaftet. Obwohl das Lamento über den Tod der Königin Isabella (Triste Espana sin ventura, CMP, Nr. 83) erst in deren Todesjahr (1504) entstanden sein kann, datiert der größte Teil seines poetischen und musikalischen Euvres gemäß eigenem Zeugnis aus den Jahren 1492 bis 1498.

Encinas Übertragungen der Bucolica von Vergil beweisen nicht nur seine große Vertrautheit mit diesem Dichter, sondern sind zugleich der erste Versuch einer Übertragung klassischer lateinischer Verse in das Kastilische. In seinen zehn Eklogen überträgt Encina Vergils Hexameter in Achtsilber und stellt einen direkten historischen Bezug zur Herrschaft der Katholischen Könige her. Die Egloga de tres pastores zeigt zudem eine offenkundige Parallele in der Naturauffassung zu Iacopo Sannazaros Schäferroman Arcadia (entstanden 1494 und erschienen 1504), der seinen Titel

20. Gioseffo Zarlino, Le istitutioni harmoniche, Fak.-Reprint der Ausgabe Venedig 1558 (=MMMLF, Ser. 2, Vol. I). New York 1965. Hier: Cap. II, 6, S. 67. Weitere Zeugnisse zur Einheit von Poet und Komponist bei Daniel Pickering Walker, Der musikalische Humanismus im 16. und frïhen 17. Jahrhundert (=Musikwissenschaftliche Arbeiten, hrsg. v. d. Gesellschaft für Musikforschung, Nr.5). Kassel / Basel 1949. Hier: S. 10ff, sowie Claude V. Palisca, Humanism in Italian Renaissance musical thought. New Haven/London 1985. Hier: S. 369ff.

21. Vgl. E. Giménez Caballero, Hipótesis a un problema de Juan del Encina. In: Revista de Filologia Española, XIV (1927), S. 59-69. Im CMP findet sich ein anonymer Villancico mir dem Textanfang „So ell enzina, enzina“, Nr. 20.

22. Zur Biographie Encinas vgl. Henry W. Sullivan, Juan del Encina. Boston 1976. Dort auch die gesamte ältere Literatur. Im Hinblick auf die Musik findet sich die gegenwärtig eingehendste Darstellung bei Robert Stevenson, Spanish Music in the Age of Columbus. Den Haag 1960, S. 253-272. Vgl. auch Miguel Querol, La produción musical de Juan del Encina (14691529). In: AnM 24 (1969), S. 121-131.

23. C.V. Palisca, a. a. O., Fußnote 21, S. 338ff. 
Vergil entlehnt. ${ }^{24}$ Die Eklogen haben im wesentlichen Encinas Ruhm als „Vater des spanischen Theaters" begründet. Diese Wertschätzung hatte aber wohl auch zur Folge, daß seine Bedeutung als Lyriker und Komponist weniger wahrgenommen wurde.

Bemerkenswerterweise ist von Encina keine geistliche Musik überliefert, obwohl er als Kind dem Chor der Kathedrale von Salamanca angehörte, dort im Jahre 1490 - als er das Alter erreicht hatte, um die niederen Weihen zu empfangen - zum , capellán de coro ‘ ernannt wurde und sich im Jahr des Erscheinens des CMP um die Stelle eines Sängers im Chor von Salamanca bewarb, die die Option auf einen Kapellmeisterposten beinhaltete. Für die Zeit seines Ende 1499 beginnenden langjährigen Aufenthaltes am päpstlichen Hof in Rom fehlt in den Quellen jeder Hinweis, der auf ein kirchenmusikalisches Amt schließen ließe. Stattdessen ist eine Aufführung seiner Égloga de Plácida e Vittoriano im Palast des spanischen Erzbischofs Jacopo Serra bekannt, die am 6. Januar 1513 im Beisein von Julius II., Federico Gonzaga und dem spanischen Botschafter stattfand. ${ }^{25}$ Die aus diesem Anlaß aufgeführte Ekloge wurde 1514 in Rom gedruckt und fand später noch das ausdrückliche Wohlgefallen Leos X. Bemerkenswert ist neben der hierin zum Ausdruck kommenden Wertschätzung des Papstes für Encina und der Aufführung eines spanischen Stückes in Rom vor allem die Tatsache, daß man das Hochfest der Epiphania Domini mit einer theatralischen Aufführung feierte.

Auch unter Berücksichtigung von möglicherweise eingetreten Quellenverlusten bleibt das Fehlen von geistlicher Musik des erst 1519 im Alter von fünfzig Jahren zum Priester geweihten Encina zusätzlich insofern bedeutsam, als eine Tendenz zur ausschließlichen Beschränkung auf die Komposition eigener Texte, und das heißt, da Encina keine lateinischen Werke hinterlassen hat, eben volkssprachlicher Texte, nicht zu übersehen ist. Dank der Patronage mehrerer aufeinanderfolgender Päpste ein mit Pfründen reich ausgestatteter Kleriker und somit nicht auf den Gelderwerb durch sein Schaffen angewiesen, verstand er sein Künstlertum womöglich nicht im Sinne der von Christoph Strosetzki gebrauchten Kategorie von „Literatur als Beruf“ zum Zwecke des Broterwerbes, sondern sah in seinem Schöpfertum seine Hauptaufgabe. Bedenkt man die Situation Heinrich Isaaks als Hofkapellmeister Kaiser Maximilians I., für den das Komponieren zur Hauptbeschäftigung geworden war, eine Annahme, die sich in die humanistische Vorstellung vom durch die Erzeugnisse seines Ingeniums Nachruhm erlangenden Künstlers hervorragend einfügt.

\section{Der Cancionero Musical de Palacio}

Die weltliche Polyphonie aus der Regierungszeit von Isabella und Ferdinand (1474-1516) ist im wesentlichen in fünf iberischen Cancioneros überliefert. Die nach Art und Umfang wichtigste Sammlung ist der CMP. Zusammengestellt in den Jahren um 1505 bis 1520 enthält der CMP Werke, deren Entstehungszeit einen Zeitraum von nahezu achtzig Jahren umfaßt. Von den 458 Einträgen sind

24. Vgl. Henry W. Sullivan, a. a. O., Fußnote 23, S. 106-111. Vgl. auch Royston O. Jones, Juan del Encina and renaissance Lyric Poetry. In: Studia Iberica. Festschrift fïr Hans Flasche, hrsg. v. Karl-Hermann Körner. Bern und München 1973 , S. 307-318.

25. Vgl. Alessandro Luzio, Federico Gonzaga, ostaggio alla Corte di Giulio II. In: Archivio della R. Società Romana di Storia Patria IX, iii-iv (1886), S. 550. 
zehn italienischen Ursprungs, wobei sechs Konkordanzen mit Petruccis Frottole-Drucken von 1504 (RISM 1504 ${ }^{4}$ ), 1505 (RISM 1505 ${ }^{3}$ ) und 1507 (RISM 1507 ${ }^{1}$ ) begegnen. ${ }^{26}$ Sowohl im Hinblick auf die Lebenssphäre der im CMP vertretenen Dichter und Komponisten als auch was Haltung und Stil der Werke betrifft, handelt es sich um eine im engeren Sinne höfische Sammlung. Die bedeutendsten spanischen Dichter des 15. Jahrhunderts - der Marqués de Santillana, Juan de Mena, Juan Rodriguez de Padrón, Jorge Manrique u.a. - sind hier vertreten. Während die älteren Einträge, von denen die wichtigsten von Francisco de la Torre (15 Eintragungen, darunter die berühmte instrumentale Alta, CMP Nr. 321) stammen, sich durch eine strikte Handhabung der Form der Cancion auszeichnen, bevorzugen die in die Zeit nach 1490 datierbaren Werke die offenere Form des Villancico. Mit 63 eindeutig zuweisbaren und 17 möglicherweise von ihm stammenden Werken ist Juan del Encina die dominierende Figur des CMP. Da er niemals Mitglied der Hofkapellen Isabellas und Ferdinands war und sich zum Zeitpunkt der Kompilation der Sammlung bereits in Rom aufhielt, muß die sich derart dokumentierende Wertschätzung durch den oder die Auftraggeber der Sammlung sich zu allererst auf seinen Ruhm als Künstler gestützt haben. Die Protektion, die er am päpstlichen Hof in Rom offenbar genoß und die in seinem Heimatland bekannt war, ${ }^{27}$ wird sein Prestige als Künstler weiter gesteigert haben.

Aus der Tatsache, daß der CMP als repräsentativ für den am Hofe der Katholischen Könige herrschenden Geschmack angesehen werden kann, folgt die Erkenntnis, daß die Hofkapellen von Aragon und Kastilien in der 2. Hälfte des 15. Jahrhunderts in größerer Unabhängigkeit von französischen und franko-flämischen Vorbildern existierten als etwa vergleichbare Einrichtungen in Italien.

Der einzige fremde Musiker in einer leitenden Position am Hof der Katholischen Könige war Johannes Wreede (Urreda), der aus Flandern stammte. ${ }^{28}$ Seine Kompositionen waren für die spanischen Musiker ein naheliegenderes Muster als etwa die Werke Ockeghems. In der Liste der Kapellmitglieder aus dem Jahre 1477 ist „maestro“ Urreda der einzige Fremde unter dreizehn Mitgliedern. Im Jahre 1498/99 fehlen Nachrichten über nichtspanische Kapellangehörige gänzlich.

Urredas geistliche und weltliche Werke unterscheiden sich deutlich von denen seiner spanischen Zeitgenossen und Kapellkollegen. Daß er in seinen weltlichen Werken einerseits den polyphonen Stil seiner franko-flämischen Heimat beibehielt und sich andererseits spanischer Dichtung annahm, führt, vor dem Hintergrund der für das Spanien der 2. Hälfte des 15. Jahrhunderts repräsentativen Werke im $C M P$, zu einer seltsam ambivalenten Haltung seiner Werke. ${ }^{29}$

Für die Verbindung des Villancico mit der italienischen Frottola ist Urredas schon bei den Zeitgenossen berühmtes Nunca fué pena mayor, das die Sammlung des CMP - womöglich eben deshalb nicht zufällig - eröffnet, geradezu paradigmatisch. Bartolomeo Tromboncino (um 1470 - nach

26. Nr. 78 (lib. v, 1505), 84 (lib. i, 1504), 91 (lib. vii, 1507), 98 (lib. vi, 1505 [= 1506]), 105 (lib. iii, 1504 [= 1505]) und 190 (lib. ii, 1504 [= 1505]) Vgl. auch R. Stevenson, a. a. O., Fußnote 23, S. 202.

27. Ohne bis dahin Priester zu sein, verliehen ihm Alexander VI. und Julius II. einige der einträglichsten Pfründen seines Heimatlandes. Vgl. R. Stevenson, a. a. O., Fußnote 23, S. 256f. Diese äußeren Zeichen seines Erfolges sind von ebenso großer Bedeutung wie die gewiß aus Rom eintreffenden Nachrichten über seinen literarischen Ruhm.

28. Vgl. R. Strohm, a. a. O., Fußnote 1, S. 578.

29. Vgl. Isabel Pope, Art. Urreda. In: NGD (1980); Bd. 19, S. 467-468. Hier: S. 468. 
1535) benutzt die ersten beiden Zeilen des von García Álvarez de Toledo, erstem Herzog von Alba (gestorben 1488) verfaßten Gedichtes, und verwendet Urredas Musik in den ersten drei Takten seiner Barzaletta, um dann aber in eigenständiger Art und Weise fortzufahren.

Als weiteren Beleg für diesen italienisch-spanischen Austausch sind die bereits erwähnten zehn Frottole im CMP ebenso anzuführen wie die Tatsache, daß Petrucci in seine Drucke Villancicos von Encina aufnahm. ${ }^{30}$ Hierbei ist der genannte Austausch stets in beide Richtungen zu denken. Insbesondere im für diese Aufgabe prädestinierten Neapel, wo die aragonischen Herrscher seit Beginn des 15. Jahrhunderts spanische Poeten und Musiker an ihrem Hof unterhielten und diese in Kontakt mit italienischen Dichtern wie Serafino, Sannazaro u.a. kamen, können Modelle spanischer Musiker auch wichtige Anregungen für ihre italienischen Kollegen bedeutet haben. ${ }^{31}$

Da die Musikpflege am Hofe der Katholischen Könige in der 2. Hälfte des 15. Jahrhunderts auf einer vergleichbaren Höhe mit der anderer europäischer Höfe stand und das dort gepflegte Repertoire international ausgerichtet war, ${ }^{32}$ verwundert es um so mehr, daß die spanischen Komponisten sich offenbar den durch die Franko-Flamen dominierten internationalen Stil nur in sehr begrenzter Art und Weise aneigneten und stattdessen auf dem Gebiet der weltlichen Vokalmusik eine nationale Sondertradition begründeten. Deren Hauptcharakteristikum ist das Streben nach einer formalen Klarheit und Einfachheit der musikalischen Faktur, die von den Kunstfertigkeiten der Franko-Flamen deutlich absticht. ${ }^{33}$

$\mathrm{Da}$ dies weniger mit der Nationalität des Komponisten an sich zu tun hat als vielmehr mit den stilistischen Vorgaben in einem konkreten geographischen und sozialen Umfeld, zeigt das Beispiel der beiden bedeutendsten spanischen Komponisten von Kirchenmusik im 15. Jahrhundert, Penalosa und Anchieta. Der Gegensatz von Gelehrtheit und Einfachheit manifestiert sich deutlich im Euvre dieser Komponisten. Während Penalosa einen Posten in der Päpstlichen Kapelle in Rom innehatte, verließ Anchieta sein Vaterland kaum und bekleidete dort wichtige Positionen. ${ }^{34}$

Die ins Auge fallende Einfachheit der weltlichen Poyphonie Spaniens ist von einer unter dem Einfluß des Diktums von Curtius stehenden Musikwissenschaft häufig als Zurückgebliebenheit und mangelnde Fähigkeit zur künstlerischen Adaption der als Stilmaßstab verstandenen Kompositionstechnik der Franko-Flamen interpretiert worden, wohingegen eine den nationalen Aspekt betonende spanische Musikgeschichtsschreibung im 19. Jahrhundert und der ersten Hälfte des 20. Jahrhunderts um den Nachweis einer, so nicht existierenden, Gleichberechtigung mit den Entwicklungen in Burgund und Italien bemüht war. ${ }^{35}$ Vielmehr dürfte es sich um einen im 15. Jahrhundert beginnenden

30. Vgl. R. Strohm, a. a. O., Fußnote 1, S. 580.

31. Ebd., S. 574.

32. Vgl. Higino Anglés, Die spanische Liedkunst im 15. und am Anfang des 16. Jahrhunderts. In: Higino Anglés, Scripta musicologica, hrsg. v. José López-Calo, 3 Bde., Rom 1975/6. Bd. 3, S. 1393-1400. Hier: S. 1394. Vgl. auch Francisco de P. Baldelló, La música en la casa de los Reyes de Aragón. In: AnM 11 (1956), S. 37-51. Vgl. für das 14. und frühe 15. Jahrhundert auch Maria Carmen Gómez-Muntané, La música en la Casa Real Catalano-Aragonesa durante los anos 1336-1437. 2 Bde., Barcelona 1979.

33. Ebda., S. 1394.

34. Vgl. Robert Stevenson, Art. Spain. In: NGD (1980), Bd. 17, S. 784-814. Hier: S. 786.

35. Vgl. Ludwig Finscher, Die nationalen Komponenten in der Musik der ersten Hälfte des 16. Jahrhunderts. In: Bericht über den neunten internationalen Kongress Salzburg 1964 (Internationale Gesellschaft für Musikwissenschaft), vorgelegt von Franz Giegling, Bd. 1. Kassel etc. 1964, S. 37-45. 
und sich im 16. Jahrhundert fortsetzenden Prozeß der Verselbständigung gegenüber den allgemeinen europäischen Entwicklungen handeln. ${ }^{36}$

Die Bedeutung der Folklore als Agens für die weitere Entwicklung vieler Gattungen ist kaum zu bestreiten. So wie am Ende des 15. Jahrhunderts das Volkslied in die Chanson Eingang fand und Knud Jeppesen mehr als 300 volkstümliche Singweisen in den mehrstimmigen Frottole nachweisen konnte, ${ }^{37}$ so ist auch im Hinblick auf den Villancico ein derartiger Einfluß anzunehmen. Insbesondere Marius Schneider hat sich, ausgehend vom Begriff des „Musizierens in Gestalten““38 um den Nachweis von Einflüssen der Volksmusik Spaniens auf die Kunstmusik bemüht. Beachtenswert ist vor allem sein Hinweis auf die dem Volkslied entstammenden asymmetrischen Teilungen, die sich einer schematischen Wiedergabe in Folgen von 3/4 und 4/4 Takten entziehen und durch die sich ,die meisten Widersprüche von Wort- und Tonakzent völlig beheben“ lassen. ${ }^{39}$

Die weitere Untersuchung etwa des stets gemutmaßten, aber bislang noch nicht nachgewiesenen arabischen Einflusses auf die spanische Volks- und Kunstmusik stellt gewiß eine wichtige Aufgabe für zukünftige gemeinsame Forschungen von Musikhistorikern und -ethnologen dar. $^{40}$

Im ungefähr aus dem Jahre 1525 stammenden originalen Index des CMP wird unter einem Villancico jedes spanische Lied verstanden, das nicht eine Romanze ist. Und umgekehrt wird unter ,Estranbote' jedes italienisches Lied subsummiert, ohne daß ein direktes Äquivalent zum Strambotto gegeben wäre. Die Vielfalt der poetischen und musikalischen Gestaltungsmöglichkeiten, die sich unter dem Begriff Villancico fassen lassen, und der im CMP begegnende Variantenreichtum ließen sich hier nur unter der Gefahr einer groben Vereinfachung darstellen. ${ }^{41}$ Offenbar wurde das Schema im Interesse eines möglichst differenzierten Eingehens auf den konkreten Gegenstand sehr flexibel gehandhabt.

Für ein klares zeitgenössisches Bewußtsein hinsichtlich der zwischen den beiden Gattungen bestehenden Unterschiede mag die zunächst befremdend anmutende Bezeichnung ,Estrambote ' für die in CMP enthaltenen Frottole einen Hinweis zu geben. In der spanischen Literatur bezeichnen Estramboten Verse, die einer feststehenden Strophenform (Sonett) hinzugefügt werden. „Estrambótico“ bedeutet aber auch ,extravagant, fremdartig“. ${ }^{42}$ Da die erste Möglichkeit aufgrund der

36. Vgl. Klaus Hortschansky, Kapitel I: Musikleben. In: Ludwig Finscher (Hg.), Die Musik des 15. und 16. Jahrhunderts (=Neues Handbuch der Musikwissenschaft, hrsg. v. Carl Dahlhaus, Bd. 3). Laaber 1990, S. 23-128. Hier: S. 55.

37. Vgl. Knud Jeppesen, La Frottola, 3 Bde., Bd. 3: Frottola und Volkslied: Zur musikalischen Überlieferung des folkloristischen Guts in der Frottola (=Acta Jutlandica XLII:1). Aarhus und Kopenhagen 1970, S. 13.

38. Vgl. Marius Schneider, Gestaltimitation als Kompositionsprinzip im Cancionero de Palacio. In: Die Musikforschung $X I$ (1958), S. 415-422. Hier: S. 417.

39. Vgl. Marius Schneider, Studien zur Rhythmik im Cancionero de Palacio. In: Miscelánea en homenaje a Monsenor Higino Anglés, 2 Bde. Barcelona 1958-1961, Bd. 2, S. 833-841. Hier S. 833f.

40. Vgl. Hierzu Gilbert Chase, a. a. O., Fußnote 18, S. 426: „As a poetic form it (der Villancico, Anm. des Verf.) appears to have been derived from a Spanish-Arabic verse-type known as zajal, in which the refrain came at the beginning and was repeated after every stanza." Vgl. auch Gertraut Haberkamp, Die weltliche Vokalmusik in Spanien um 1500, der Cancionero musical de Colombina von Sevilla und auß.3erspanische Handschriften (=Münchner Veröffentlichungen zur Musikgeschichte, Bd. 12). Tutzing 1968. Haberkamp nimmt insbesondere in Fragen des Instrumentenbaus und der Vortagsweise einen arabischen Einfluß an. Vgl. S. 13.

S. 196.

41. Vgl. Isabel Pope, Musical and metrical form of the villancico. In: Annales musicologiques 2 (1954), S. 189-214. Hier:

42. Vgl. G. Haberkamp, a. a. O., Fußnote 41, S. 61. 
divergierenden Form ausscheidet, erscheint es zulässig davon auszugehen, daß derjenige, der den zeitgenössischen Index erstellte, die Andersartigkeit der Frottolen bewußt wahrnahm und durch die Bezeichnung als ,,außergewöhnlich“ auch zum Ausdruck bringen wollte.

Bereits einem ersten Blick auf die musikalische Faktur der in CMP enthaltenen Frottole verdeutlichen sich diese ,Fremdartigkeiten“. So überwiegt beim Villancico die Dreistimmigkeit, bei den Frottole die Vierstimmigkeit. Ferner zeigt sich in der Frottola das Bestreben, klar zu gliedern, und zu diesem Zweck die Verse einer strengen musikalischen Gliederung zu unterwerfen und dasselbe rhythmische Schema beizubehalten, während die spanischen Fassungen der Frottola den Text mehr berücksichtigen und Schematismen zu vermeiden suchen. Auffallend ist die häufigere Verwendung von Dissonanzen in den Frottole, wogegen sie im Villancico kaum oder gar nicht begegnen. Im Gegensatz zum Villancico bringt die Frottola ferner in der Kadenz stets die Terz. Allgemein sind die Stimmen der spanischen Stücke selbständiger und nicht lediglich begleitend wie häufig in der Frottola.

Übereinstimmung herrscht zwischen den beiden Gattungen hinsichtlich der Dominanz der Oberstimme. Gemeinsamkeit herrscht weiterhin im fast gänzlichen Fehlen dreizeitiger Mensuren, dem geringen Ambitus der Oberstimme und den Schlüssen im Abstand einer Dezime. ${ }^{43}$

\section{Musik auf den Fall Granadas: Juan del Encinas Levanta, Pascual}

In der zeitgenössischen Literatur fand der Fall Granadas zunächst eine stärkere Resonanz als die Entdeckungen des Kolumbus. In Briefen der beiden als Lehrer in Spanien tätigen italienischen Humanisten Lucius Marinaeus Siculus und Petrus Martyr Anglerius, in Prunkreden, Festspielen und Berichten wurden die Taten der Katholischen Könige im ,annus mirabilis“ ihrer Herrschaft verherrlicht. Während die italienischen Autoren in epischen und halbdramatischen Werken die Ereignisse poetisch-rhetorisch überhöht darstellen und das Herrscherlob singen, widmen sich die Spanier vorwiegend der Geschichtsschreibung. ${ }^{44}$

$\mathrm{Zu}$ den für die Frühgeschichte des Humanistendramas aufschlußreichen panegyrischen Dichtungen gehört die neulateinische Historia Baetica des päpstlichen Kammerherrn Carolus Veradus (1440-1500) aus Cesena, die gleichzeitig entstand mit den beiden italienischen Schauspielen des aus einer alten spanischen Familie stammenden Dichters Iacopo Sannazaro, La presa di Granata und $I l$ triunfo de la Fama, die am neapolitanischen Hofe des Duque Alfonso de Calabria aufgeführt wurden (4. bzw. 6. März 1492). ${ }^{45}$

Wolfgang Osthoff stellt den 1493 in Rom am Schluß der Historica Baetica abgedruckten vierstimmigen Triumphgesang Viva el gran Re Don Fernando an den Ausgangspunkt seiner Untersuchungen zu Theatergesang und darstellender Musik in der italienischen Renaissance. ${ }^{46}$ In

43. Ebda., S. $62 \mathrm{f}$.

44. Vgl. Dietrich Briesemeister, Episch-dramatische Humanistendichtungen zur Eroberung von Granada (1492). In: Texte-Kontexte-Strukturen: Festschrift K.A. Blïher, hrsg. v. A. de Toro, Tübingen 1987, S. 249-263. Hier: S. 249.

45. Zu Sannazaros Farsen vgl. Marvin T. Herrick, Italian Comedy in the Renaissance. Urbana 1960, S. 26-29.

46. Vgl. Wolfgang Osthoff, Theatergesang und darstellende Musik in der italienischen Renaissance (15. und 16. Jahrhundert) (=Münchner Veröffentlichungen zur Musikgeschichte, Bd. 14). Tutzing 1969, S. 15-29. 
Anlehnung an Alfred Einstein ${ }^{47}$ spricht Osthoff von einem der ersten gedruckten Beispiele der Frottola und konkreter von einer Barzaletta, die durch die ausschließliche Verwendung des Ottonario charakterisiert ist. ${ }^{48}$

$$
\text { Reim Teil: }
$$

Viva el gran Re Don Fernando

Con la Reyna Don Isabellá

Viva Spagna e la Castella

Pien de gloria triumphando

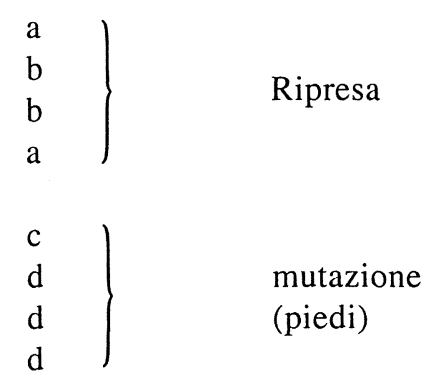

La Cita Mahomectana

Potentissima Granata

Da la falsa fe pagana

E dissolta e liberata

Per virtute \& manu armata

Del Fernando e Lisabella

$\left.\begin{array}{l}\mathrm{d} \\ \mathrm{b}\end{array}\right\} \quad$ volta

Viva Spagna e la Castella

Pien de gloria triumphando

$\left.\begin{array}{l}\mathrm{b} \\ \mathrm{a}\end{array}\right\} \quad$ Ripresa (2. Hälfte)

47. Alfred Einstein, The Italian Madrigal, Vol. 1. Princeton 1949, S. $35 \mathrm{ff}$.

48. Reimschema aus W. Osthoff, a. a. O., Fußnote 47, S. 16. 


\section{Viva el gran Rey Don Fernando ${ }^{49}$}

Carlo Verardi, 1492
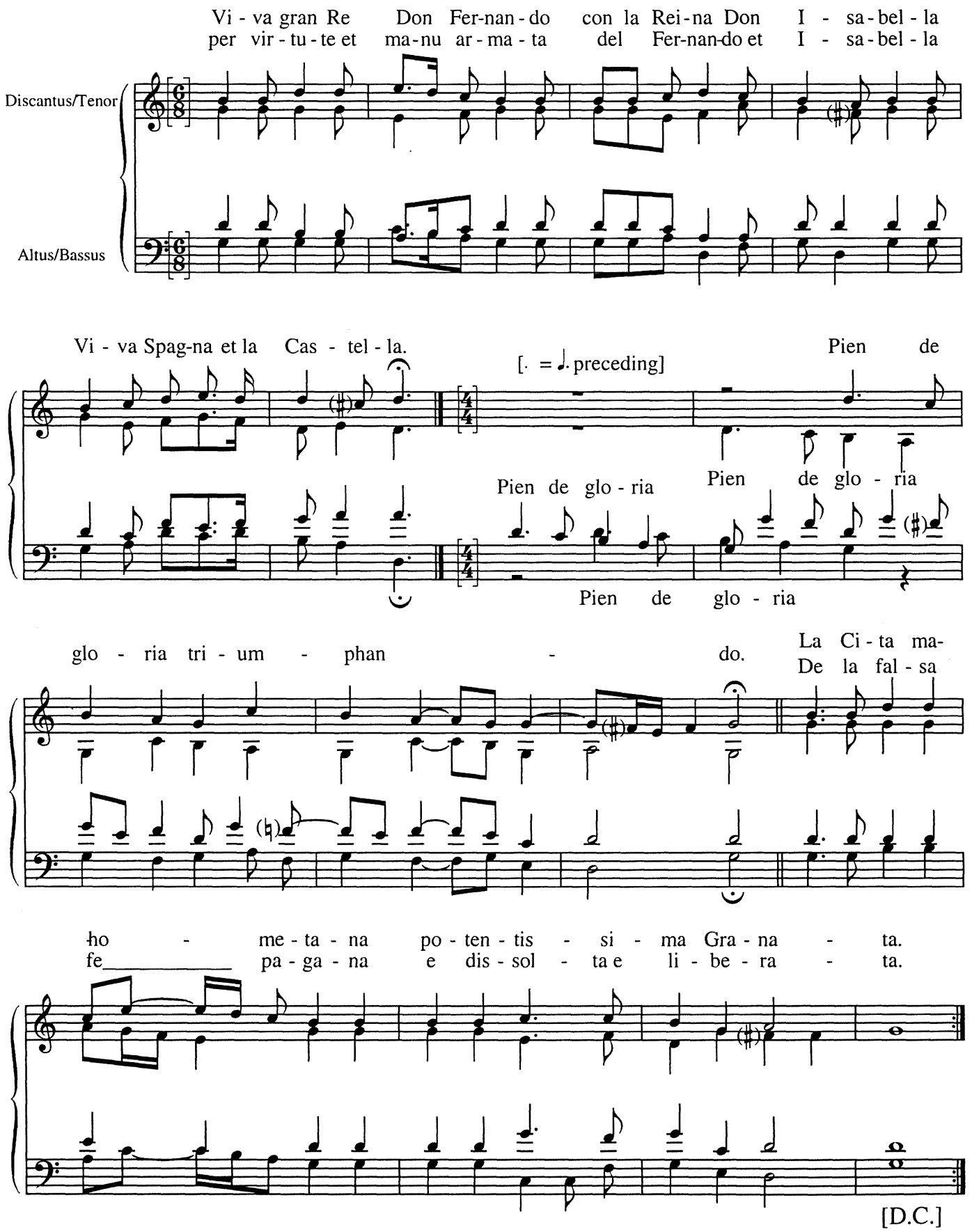

49. Notenbeispiel abgedruckt nach W. Osthoff, a. a. O., Fußnote 47, Notenteil, S. 135-137. 
Da die Anordnung in der gedruckten Quelle einen direkten Bezug von Viva el gran Re Don Fernando auf die Aufführung der Historia Baetica nicht erkennen läßt und auch keine dokumentarische Evidenz für eine solche Annahme gegeben ist, bezweifelt Osthoff zu Recht die Zugehörigkeit zur Historia Baetica, spricht sich aber auch gegen Einsteins Annahme einer Aufführung im Zusammenhang mit Sannazaros Il triunfo de la Fama in Neapel aus. Stattdessen glaubt er aufgrund eher vager stilkritischer Mutmaßungen eine Florentiner Provenienz des Gesanges postulieren zu können. ${ }^{50}$

Betrachtet man Viva el gran Re Don Fernando jedoch im Hinblick auf die mögliche Nähe zu Gestaltungsprinzipien einer spanischen Gattung, so stellt man unschwer fest, daß Viva el gran Re Don Fernando dichterisch und musikalisch der Form der Cancion, der höchstentwickelten geschlossenen Form der kastilischen Poesie, in vielen Punkten entspricht: ${ }^{51}$

\begin{tabular}{|c|c|c|c|c|c|}
\hline Music & & & Music & & erse \\
\hline A & $\begin{array}{l}\mathrm{a} \\
\mathrm{b} \\
\mathrm{b} \\
\mathrm{a}\end{array}$ & $\left\{\begin{array}{l}a \\
b \\
a \\
b\end{array}\right.$ & $\mathbf{A}$ & $\begin{array}{l}a \\
b \\
a \\
a \\
b\end{array}$ & $\left\{\begin{array}{l}a \\
b \\
a \\
a \\
b\end{array}\right.$ \\
\hline B & $\begin{array}{l}\mathrm{c} \\
\mathrm{d}\end{array}$ & $\left\{\begin{array}{l}c \\
d\end{array}\right.$ & B & $\begin{array}{l}\mathrm{c} \\
\mathrm{d}\end{array}$ & $\left\{\begin{array}{l}c \\
d\end{array}\right.$ \\
\hline B & $\begin{array}{l}\mathrm{d} \\
\mathrm{c}\end{array}$ & $\left\{\begin{array}{l}c \\
d\end{array}\right.$ & $\begin{array}{l}\text { B } \\
\text { C }\end{array}$ & $\begin{array}{l}\mathrm{c} \\
\mathrm{c} \\
\mathrm{d}\end{array}$ & $\left\{\begin{array}{l}c \\
c\end{array}\right.$ \\
\hline A & $\begin{array}{l}a \\
b \\
b \\
a\end{array}$ & $\left\{\begin{array}{l}a \\
b \\
a \\
b\end{array}\right.$ & A & $\begin{array}{l}a \\
b \\
a \\
a \\
b\end{array}$ & $\left\{\begin{array}{l}a \\
b \\
a \\
a \\
b\end{array}\right.$ \\
\hline
\end{tabular}

Aber auch ohne die Nähe von Viva el gran Re Don Fernando zur

Form der Cancion überrascht es, wenn Osthoff feststellt, daß zwischen diesem Werk und dem von ihm gleichfalls herangezogenen Villancico Levanta, Pascual „keinerlei Unterschied“ bestehe. ${ }^{52}$ Ein erster Blick auf Encinas Werk offenbart den grundlegenden Unterschied:

Levanta, Pascual, levanta,

Aballemos a Granada,

Que se suena qu'es tomada

50. „Wenn man Frottolenmusik kennt und unser Stück hört, hat man den Eindruck einer ganz anderen Haltung. Man ist an die Musik eines anderen damaligen Kulturkreises erinnert, an die Musik von Florenz." W. Osthoff, a. a. O., Fußnote 47, S. 27.

51. I. Pope, a. a. O., Fußnote 42. S. 198. Schema des Cancion nach I. Pope, a.a.O., S. 198/199.

52. W. Osthoff, a. a. O., Fußnote 47, S. 22. 
Levanta toste priado,

Toma tu perro y currón,

Tu camarra y camarrón

Tus albogues y cayado.

Vamos ver el gasajado

D'aquella ciudad nombrada,

Que se suena qu'es tomada.

\section{Levanta, Pascual ${ }^{53}$}

Juan del Encina

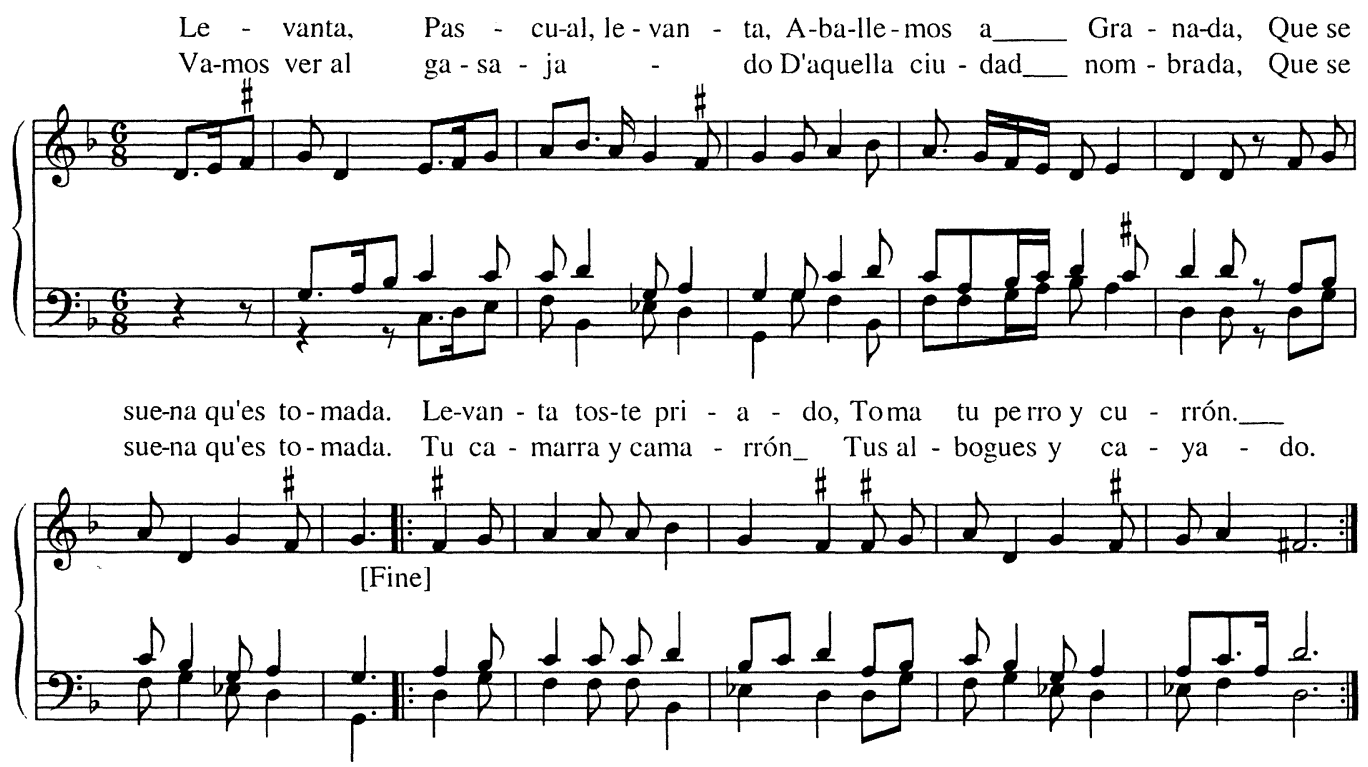

Die dichterische und musikalische Anlage entspricht bis auf die Abweichung im eröffnenden Vers, die aber in den folgenden Strophen wieder angeglichen wird, dem von Isabel Pope ${ }^{54}$ als einem der am häufigsten begegnenden Formen des Villancico im CMP und darüber hinaus bezeichneten Schema:

53. Notenbeispiel abgedruckt nach R. Stevenson, a.a.O., Fußnote 23, S. 267.

54. Schema abgedruckt nach I. Pope, a.a.O., Fußnote 42, S. 197/4. 


$\left.\begin{array}{lll}\text { Estribillo } & \left\{\begin{array}{l}\mathrm{a} \\ \mathrm{b}\end{array}\right. & \mathrm{a} \\ \mathrm{b} & \mathrm{b} \\ \text { Mudanza I } & \left\{\begin{array}{l}\mathrm{c} \\ \mathrm{d}\end{array}\right. & \mathrm{A} \\ \text { Mudanza II } & \left\{\begin{array}{l}\mathrm{d} \\ \mathrm{c}\end{array}\right. & \mathrm{e} \\ \text { Vuelta } & \left\{\begin{array}{l}\mathrm{c} \\ \mathrm{b}\end{array}\right. & \mathrm{e} \\ \mathrm{b} & \mathrm{d} \\ \mathrm{b}\end{array}\right\}$

Die auf denselben historischen Gegenstand Bezug nehmenden Kompositionen zeigen somit ein sehr verschiedenes Gepräge, und dies sowohl hinsichtlich der dichterischen als auch der musikalischen Gestaltung. Die gezeigte Nähe von Viva el gran Re Don Fernando zur Form der Cancion scheint zudem Einsteins Vermutung einer Zugehörigket des Werkes zu einer Aufführung von Sannazaros Il triunfo de la Fama zu bestätigen. Dies umso mehr, als Jeremy N. H. Lawrance die reiche Tradition und Dichte der Aufführung von italienischen Farsen am aragonesischen Hof in Neapel nachgewiesen hat, ${ }^{55}$ die es in einer derartigen Dichte in Rom nicht gegeben hat. Eine derartige panegyrische Akklamation beschloß zumeist die Aufführungen der Stücke, wie auch in den Eklogen Encinas am Ende ein Villancico erklang. Hingewiesen sei zudem auf die Cancion Viva, Viva rey Ferrando (Monte Cassino. Biblioteca dell'Abbazia. MS 871, olim 871n), ${ }^{56}$ die, auf Ferdinand I. von Aragon bezogen, doch eine historische Parallelerscheinung am selben Ort darstellt, wobei dann die Wahl der Form für Viva el gran Re Don Fernando im Anschluß an lokale Traditionen erfolgt wäre.

Die gravierendsten Unterschiede zwischen den beiden Werken dokumentieren sich jedoch nicht auf der Ebene der dichterischen Form oder des musikalischen Satzes, sondern im unterschiedlichen Umgang mit dem das konkrete Werk hervorrufenden historischen Ereignis. Während sich Viva el gran Re Don Fernando in den traditionellen Bahnen höfischer Panegyrik bewegt - und dies zeigt nichts deutlicher als der Vergleich mit der etwa dreissig Jahre älteren Canción Viva, viva rey Ferrando - , so siedelt Encina seine Auseinandersetzung mit dem Fall von Granada in einer deutlich den Einfluß von Vergil verratenden arkadischen Hirtenidylle an; daß dies als Inbegriff der zu seiner Zeit modernsten humanistischen Bestrebungen gelten darf, wurde oben gezeigt.

Dies verkennt nicht, daß der Panegyriker am Hof in Neapel - womöglich der mit den spanischen Formen vertraute Sannazaro selbst - sich den aus dem Anlaß einer hochoffiziellen Aufführung resultierenden Anforderungen an den Grad der Repräsentativität nicht zu entziehen vermochte und Encina, am Hof der Herzöge von Alba sich in einer eher privaten Atmosphäre

55. J. N. H. Lawrance, a.a. O., Fußnote 6, S. 234 f. 41, S. $284-287$.

56. Anders als von Osthoff behauptet, handelt es sich nicht um die gleichen Verse, vgl. G. Haberkamp, a. a. O., Fußnote 
bewegend, größeren Gestaltungsspielraum besessen haben mag. Dies setzt jedoch nicht die Tatsache außer Kraft, daß Encina die modernere künstlerische Anverwandlung des historischen Geschehens bietet, ohne jede Spur von „Verspätung“.

Der bedeutendste spanische Humanist des 16. Jahrhunderts, Juan Luis Vives, entdeckt im Ingenium die Erfindungsgabe des Menschen für den schöpferischen Aufbau der historischen Welt. Ohne die ingeniöse Tätigkeit bleibt dem Menschen die eigentliche Funktion des Wortes und der Erkenntnis verschleiert. ${ }^{57}$

Im Bezug Encinas in Levanta, Pascual auf das bedeutendste Ereignis der spanischen Geschichte des 15. Jahrhunderts dokumentiert sich ein schöpferisches Selbstverständnis und ein Bewußtsein für die Bedeutsamkeit und Eigenständigleit der spanischen Geschichte, die einen Vergleich mit dem zeitgenössischen italienischen Humanismus nicht zu scheuen braucht.

57. Vgl. Emilio Hidalgo-Serna, Einleitung, in: Juan Luis Vives, Über die Gründe des Verfalls der Künste. De Causis corruptarum artium. Lateinisch-deutsche Ausgabe, hrsg. v. Emilio Hidalgo-Serna (=Humanistische Bibliothek, Reihe 2/28). München 1990, S. 7-99. Hier: S. 36. 